\title{
Left atrial booster-pump function as a predictive parameter for atrial fibrillation in patients with severely dilated left atrium
}

\author{
Chia-Hung Yang ${ }^{1,2}$, Hao-Tien Liu ${ }^{1}$, Hui-Ling Lee ${ }^{3}$, Fen-Chiung Lin $^{1}$, Chung-Chuan Chou ${ }^{1,4} \wedge$ \\ ${ }^{1}$ Division of Cardiology, Department of Internal Medicine, Chang Gung Memorial Hospital, Linkou Branch, Taoyuan; ${ }^{2}$ Department of Internal \\ Medicine, New Taipei Municipal TuCheng Hospital, New Taipei; ${ }^{3}$ Department of Anesthesia, Chang Gung Memorial Hospital, Taipei Branch, \\ Taipei; ${ }^{4}$ Chang Gung University College of Medicine, Taoyuan
}

Contributions: (I) Conception and design: CC Chou, FC Lin; (II) Administrative support: CC Chou; (III) Provision of study materials or patients: CC Chou, CH Yang; (IV) Collection and assembly of data: HL Lee, CC Chou; (V) Data analysis and interpretation: CH Yang, HT Liu, HL Lee, CC Chou; (VI) Manuscript writing: All authors; (VII) Final approval of manuscript: All authors.

Correspondence to: Chung-Chuan Chou, MD. Chang Gung Memorial Hospital, Linkou Branch, 5 Fu-Xing Street, Guishan District, Taoyuan 33305. Email: 2867@adm.cgmh.org.tw.

Background: Left atrial (LA) dimension $\geq 50 \mathrm{~mm}$ had approximately four times the risk of developing atrial fibrillation (AF). The aim of this study was to investigate whether the application of clinical and echocardiographic parameters could differentiate between the patients having severely dilated left atrium with and without AF.

Methods: This retrospective cross-sectional study enrolled consecutive patients with LA dimension $\geq 50$ $\mathrm{mm}$ and divided them into three groups: no AF (no-AF), paroxysmal AF (PAF) and non-paroxysmal AF (non-PAF) groups. For PAF and non-PAF groups, all patients underwent radiofrequency ablation, and the echocardiographic parameters were obtained on the next day after ablation.

Results: Our study population comprised 160 patients, including 80, 53, and 27 patients in the non-AF, $\mathrm{PAF}$ and non-PAF groups, respectively. The no-AF group had a significantly higher body mass index $\left(\mathrm{kg} / \mathrm{m}^{2}\right)$ $(29.31 \pm 6.27,27.58 \pm 4.12$ and $26.57 \pm 2.81, \mathrm{P}=0.01)$, and a higher prevalence of diabetes mellitus (DM) [31 (38.80\%), $13(25.00 \%)$ and 4 (14.80\%), P=0.01] and hypertension [67 (83.80\%), $34(65.40 \%)$, and $19(70.40 \%), \mathrm{P}=0.04]$, but a lower prevalence of rheumatic heart disease (RHD) [3 (3.80\%), $6(11.50 \%)$ and $5(18.50 \%), \mathrm{P}=0.02]$ and sick sinus syndrome [0 (0.00\%), $6(11.50 \%)$ and $4(14.80 \%), \mathrm{P}=0.045]$. Echocardiographic studies showed that the non-AF group had significantly smaller LA minimal volume index $\left(24.89 \pm 9.74,34.06 \pm 19.38\right.$ and $\left.42.83 \pm 17.44 \mathrm{~mL} / \mathrm{m}^{2}, \mathrm{P}<0.01\right)$, higher LA emptying fraction $(51.99 \% \pm 13.97 \%$, $38.40 \% \pm 15.96 \%$ and $33.89 \% \pm 10.73 \%, \mathrm{P}<0.01)$, longitudinal strain $(23.87 \% \pm 7.72 \%, 17.11 \% \pm 8.52 \%$ and $12.38 \% \pm 4.28 \%, \mathrm{P}<0.01)$ and strain rate than the $\mathrm{AF}$ groups. The multivariate analysis showed that the late diastolic component of LA strain rate was the only independent factor associated with the presence of AF (odds ratio, 21.69; 95\% CI, 9.77-48.13, $\mathrm{P}<0.01)$.

Conclusions: LA function plays an important role in the absence of AF in patients with LA dimension $\geq 50 \mathrm{~mm}$; the late diastolic component of LA strain rate was the only independent variable on multivariate analysis.

Keywords: Atrial fibrillation (AF); left atrial dilatation; speckle tracking echocardiography

Submitted Sep 27, 2021. Accepted for publication Jan 12, 2022.

doi: 10.21037/qims-21-954

View this article at: https://dx.doi.org/10.21037/qims-21-954

\footnotetext{
^ ORCID: 0000-0003-4465-0016.
} 


\section{Introduction}

Atrial fibrillation (AF) is the most common arrhythmia in daily clinical practice worldwide. It has a potential risk to cause ischemic stroke, leading to severe neurologic sequela and even death $(1,2)$. Left atrial (LA) dimension (LAD) in M-mode unidimensional measurement has been shown to predict $\mathrm{AF}$ occurrence in general adult (3) and elderly populations $(2,4)$. The Cardiovascular Health Study showed that patients with sinus rhythm and the LAD larger than $50 \mathrm{~mm}$ had approximately four times the risk to develop AF during the surveillance (4). However, some patients with enlarged left atrium do not have AF. A recent metaanalysis reported that the most powerful LA predictor of AF recurrence after ablation was LA strain $<20 \%$, followed by $\mathrm{LAD} \geq 50 \mathrm{~mm}$, and LA maximal volume $>150 \mathrm{~mL}$ (5). This suggests that atrial function plays a more important role in $\mathrm{AF}$ genesis than $\mathrm{LAD}$.

There is increasing recognition that atrial fibrosis is one of the contributing abnormalities to the development of AF (6). Delay-enhancement magnetic resonance image and intracardiac electroanatomic voltage mapping were two methods suggested to identify atrial fibrosis (7), but the invasiveness and high time consumption limit the routine application of these tools in patients with LA dilatation. Echocardiography has been the imaging technique of choice for evaluating the left atrium because of its widespread availability and ease of use. Speckletracking echocardiography (STE) is a non-Doppler echocardiographic method to quantify atrial deformation by calculating both the atrial longitudinal strain and strain rate of atrial segments and has been proposed as a non-invasive technique to assess atrial fibrosis $(8,9)$. The influences of strain and strain rate on patients with $\mathrm{AF}$ were described previously (10). However, the LA mechanics in patients with severely enlarged left atrium without AF have not been well evaluated yet. The aim of this study was to investigate whether the application of clinical and echocardiographic parameters could differentiate between the patients that have severely dilated left atrium with and without AF. We present the following article in accordance with the STROBE reporting checklist (available at https://qims. amegroups.com/article/view/10.21037/qims-21-954/rc).

\section{Methods}

\section{Study population}

This retrospective cross-sectional study evaluated patients with severely dilated left atrium (LAD $\geq 50 \mathrm{~mm}$ ) between September 2014 and October 2019 at Chang Gung Memorial Hospital, Linkou. In accordance with the HRS/ EHRA/ECAS expert consensus statement, paroxysmal AF (PAF) was defined as AF that terminates spontaneously or with intervention within 7 days of onset; persistent $\mathrm{AF}$ is defined as continuous AF that is sustained beyond 7 days of onset; and long-standing persistent AF is defined as continuous AF of greater than 12 months' duration (11). We divided our patients into three groups: no-AF, PAF, and non-PAF (including persistent and long-standing persistent AF) groups. For PAF and non-PAF groups, all enrolled patients had undergone radiofrequency catheter ablation (RFCA), and the echocardiographic parameters were obtained on the next day after ablation. Patients under 20 years of age or undergoing previous valve surgery or MAZE procedure were excluded. Patients who had nonPAF had early atrial tachyarrhythmia recurrence and could not maintain sinus rhythm on the next day after RFCA were also excluded $(n=2)$. Clinical data were obtained from a comprehensive review of each patient's medical record. All identification data were encrypted before the information was released to researchers. This study was conducted in accordance with the Declaration of Helsinki (as revised in 2013). This study was approved by the Institution Review Board of Chang Gung Memorial Hospital (No. 202101298B0) and individual consent for this retrospective analysis was waived.

\section{Echocardiography}

Doppler M-mode and two-dimensional (2-D) echocardiography were performed using a commercially available ultrasonography machine (Vivid 9, General Electric Medical Health, Waukesha, WI, USA) with a $2.5-\mathrm{MHz}$ phased-array transducer. All patients were in sinus rhythm during echocardiography. For AF patients, transthoracic echocardiography was performed on the next day after ablation. All echocardiographic measurements were obtained in accordance with the guidelines of the American Society of Echocardiography (12). The LAD was assessed by measuring the anteroposterior dimension in the M-mode measurement. The 2-D LA volume was measured by the biplane area-length method from the apical 4-chamber (4-C) view. The maximal LA volume (LAVmax) was defined as the volume just before the mitral valve opening, and the minimal LA volume (LAVmin) was defined as the smallest volume during ventricular diastole. 
The LA emptying fraction (LAEF) was calculated as (LAVmax $-\mathrm{LAVmin}$ )/LAVmax $\times 100 \%$ (13). Mitral inflow was recorded between the tips of the mitral leaflets by pulsed-wave Doppler at the apical position. Peak velocities of early (E) and atrial (A) diastolic filling were measured. Color Tissue Doppler imaging was obtained in the apical 4-C view. Peak early diastolic (E') myocardial velocities were measured within a $6-\mathrm{mm}$ circular sample volume at the septal and lateral mitral annular positions. Ratios of E/E' were calculated for both the septal $\mathrm{E} / \mathrm{E}$ ' and lateral E/E'.

STE images of the left atrium obtained in apical 4-C and 2-chamber (2-C) views with a frame rate between 60 and 100 frames/s were captured and stored digitally for offline analysis of LA strain and strain rate (EchoPac PC, GE Vingmed, Horton, Norway) (13). Special care was taken during echocardiographic image acquisition to ensure adequate LA tracking and avoid interference with the pulmonary veins and LA appendage to measure LA strain and strain rate. The endocardium of the LA wall was manually traced starting from the medial/septal to the lateral mitral annulus in the apical 4-C view and from inferior to anterior mitral annulus in the apical 2-C view and was tracked by the 2-D speckle-tracking software along the border. The operator manually adjusted segments that were not tracked. STE determined regional changes in length and was expressed as a positive value for lengthening or as a negative value for shortening. Time-strain and time-strain rate plots were produced automatically by the software (EchoPAC, version R202). LA peak ventricular systolic longitudinal strain (LASr) was measured by adjusting the electrocardiography gating to the start of the QRS complex during the LA reservoir phase. The LA strain rate pattern is characterized by a positive wave occurring during ventricular systole and two negative waves during early and late diastole. The systolic component of the LA strain rate mostly reflects LA reservoir function (LASRr), whereas the early diastolic and late diastolic components mostly reflect LA conduit (LASRe) and contractile function (LASRa), respectively. All indexes were obtained by averaging values obtained in the apical 4-C and 2-C views (14). These parameters were measured for accessing longitudinal deformation of the left atrium (15).

We have previously shown that both intra- and interobserver variability of STE-derived LA functional parameters were low $(13,16)$. In this study, we evaluated the intraobserver and interobserver variability of strain and strain rate in 10 randomly selected patients, measured first by the same investigator on two separate occasions for intraobserver variability, and then by two independent investigators for interobserver variability. The two investigators were blinded to each other's measurements and the outcome status of AF ablation. Repeated measurement was made at the same cardiac cycle of the same image for each patient to avoid inherent variability caused by different cycle lengths.

\section{Electrophysiological study and RFCA}

All patients underwent RFCA under endotracheal intubation and general anesthesia. Heparin was administered to keep the activated clotting time $>300 \mathrm{~s}$. RFCA was performed using a $3 \mathrm{D}$ electroanatomical mapping system (Carto 3, Biosense Webster, Diamond Bar, CA, USA) to support the creation and validation of ablation lesions. A 3.5-mm open-tip irrigated catheter (NaviStar Thermo-Cool, Biosense Webster) was percutaneously introduced through the right femoral vein for mapping and ablation. Circumferential pulmonary vein isolation (CPVI) with confirmation of entrance block was verified in all patients. The ablation catheter was moved point by point in a dragging fashion to create successive lesions. If $\mathrm{AF}$ persisted or LA tachycardia occurred after CPVI, additional LA linear ablation was performed at the operator's discretion. As reported previously, we preferred performing linear ablation within low-voltage area, especially at sites with complex-fractionated and/or high-frequency electrograms (17). External cardioversion was performed to restore sinus rhythm if RFCA failed to convert AF. The attempted endpoint of linear ablation was conduction block, validated by differential pacing with bidirectional reversal of the peri-mitral activation sequence and/or the recording of local separated double potentials at the entire ablation line. Non-pulmonary vein triggers that reinitiated AF were ablated as deemed necessary. The patients undergoing RFCA were followed up at 1 week, 1 month, 3 months, 6 months and every 3-6 months after RFCA or whenever required because of $\mathrm{AF}$ symptoms.

\section{Statistical analysis}

Continuous variables are expressed as the mean \pm standard deviation, and categorical variables are presented by a number with a percentage in brackets. To assess the association between variables and the status of no AF, PAF, and non-PAF, we performed analysis of variance procedures with post-hoc LSD analysis for continuous variables and 
Table 1 Clinical characteristics of the study groups

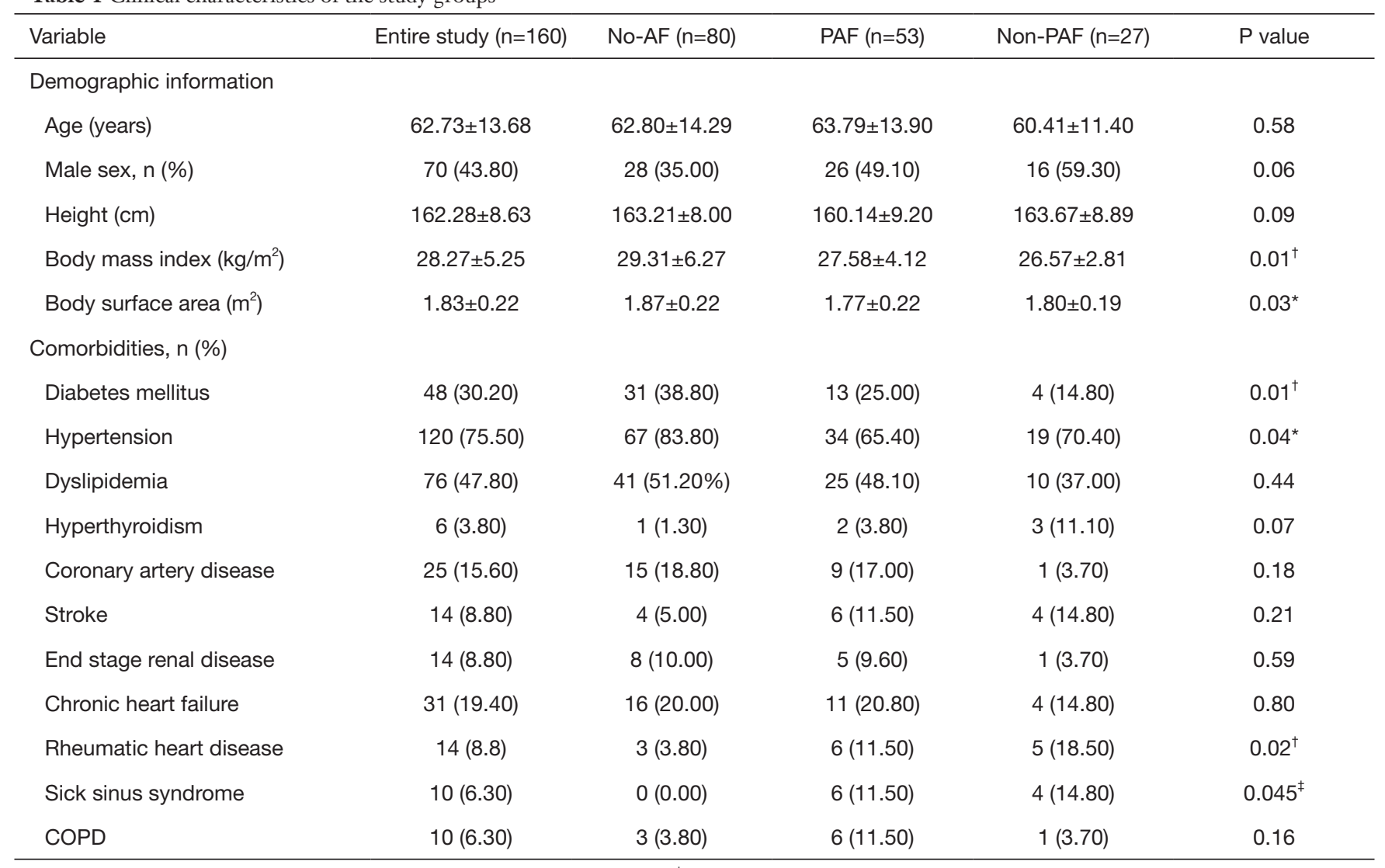

*, there was significantly different between no-AF and PAF groups; ${ }^{\dagger}$, there was significantly different between no-AF and non-PAF groups; ${ }^{\ddagger}$, there was significantly different between no-AF group and PAF group, and no-AF group and non-PAF group. AF, atrial fibrillation; COPD, chronic obstructive pulmonary disease; PAF, paroxysmal AF.

the chi-square test for categorical variables. To evaluate the effects of RFCA on LA strain and strain rate parameters, we performed repeated-measures analysis of variance with posthoc LSD analysis to analyze differences in LASr, LASRr, LASRe, and LASRa (before ablation, 1-day, and 3-month post ablation) in patients with or without additional LA linear ablation in the PAF group and paired $t$-test (1-day and 3 -month post ablation) in the non-PAF group. The beatto-beat difference of LA strain and strain rate parameters between two consecutive beats was evaluated by paired $t$-test. A proportional odds logistic regression model using a backward stepwise method (selection for entry criteria $=0.05$; selection for stay criteria $=0.1$ ) was used to identify the independent predictors (18). The odds ratio and $95 \%$ confidence interval (CI) for each variable were calculated. Variables with $\mathrm{P}<0.05$ in univariate analysis were considered in multivariate regression models.

\section{Results}

\section{Baseline characteristics and echocardiographic parameters}

Our study population comprised 160 patients (mean age $62.73 \pm 13.68$ years, $43.8 \%$ male), including 80 patients with no history of AF (after a mean follow-up of $30.4 \pm 18.5$ months), 53 patients with PAF, and 27 patients with non-PAF. There were 14 of 53 patients in the PAF group and 26 of 27 patients in the non-PAF group undergoing additional LA linear ablation. Table 1 summarizes the clinical characteristics of the study groups. The no-AF group had a significantly higher body mass index (BMI) than the PAF and non-PAF groups. Additionally, the no-AF group had a significantly higher prevalence of diabetes mellitus (DM) and hypertension but a lower prevalence of rheumatic heart disease (RHD) and sick sinus syndrome than the $\mathrm{AF}$ groups. 
Table 2 Echocardiographic characteristics of the study groups

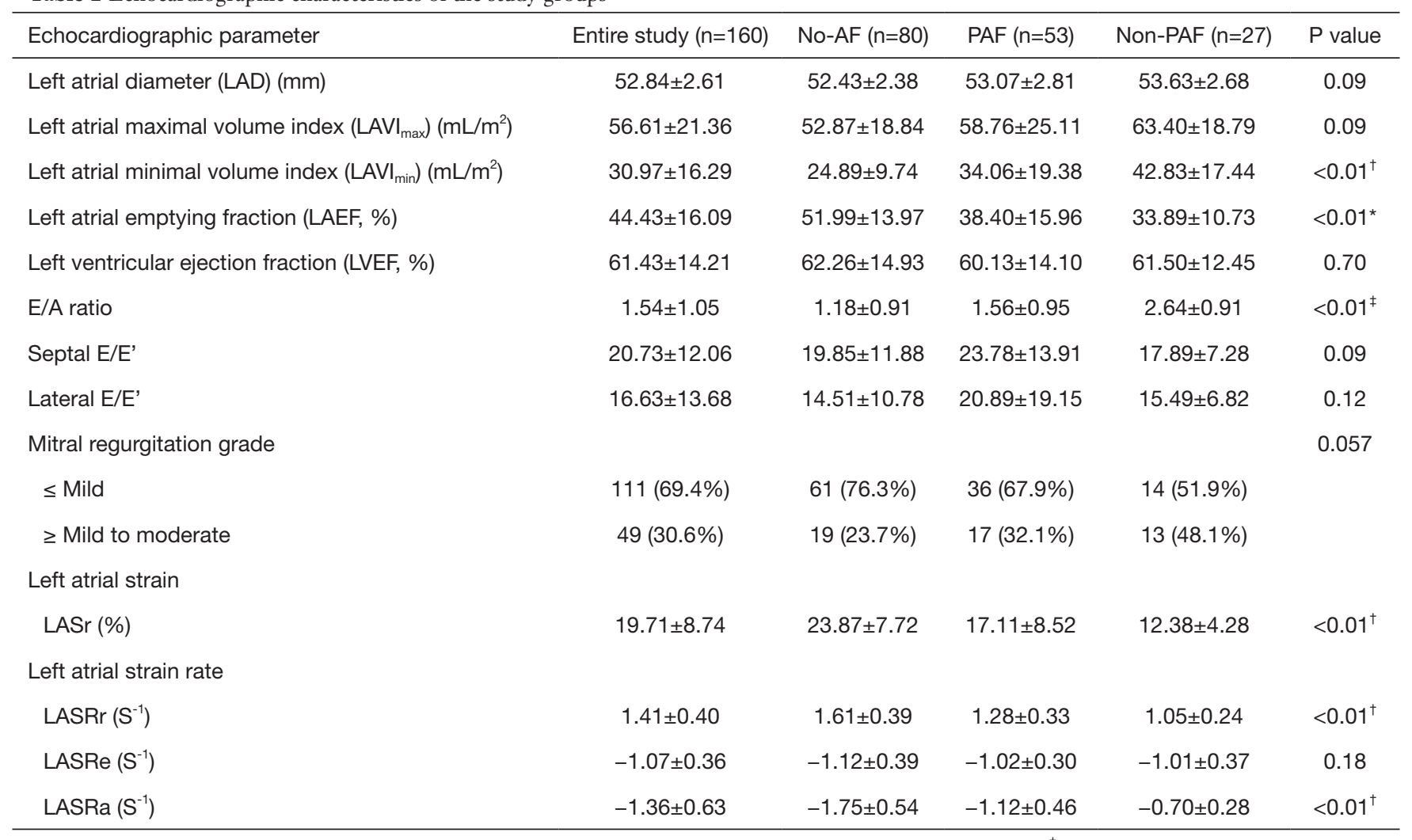

${ }^{*}$, there was significantly different between no-AF and PAF groups, and no-AF and non-PAF groups; ${ }^{\dagger}$, there was significantly different between no-AF and PAF groups, PAF and non-PAF groups, and no-AF and non-PAF groups; ${ }^{\ddagger}$, there was significantly different between no-AF and non-PAF groups, and PAF and non-PAF groups. LASr, peak positive longitudinal strain during atrial filling; LASRr, strain rate during ventricular systole as an index for left atrial reservoir function; LASRe and LASRa, peak negative conduit and contractile strain rates during left ventricular early and late refilling, respectively.

\section{Standard echocardiographic characteristics and STE parameters}

The results of comparisons of the standard echocardiographic parameters and STE-derived LA function parameters among the no-AF, PAF, and nonPAF patients are illustrated in Table 2. The patients in the no-AF group exhibited significantly smaller LAVImin, larger LAEF and smaller E/A ratio compared with patients with $\mathrm{AF}$ ( $\mathrm{P}<0.01$ for both comparisons). There was no significant difference of LAD, LAVImax, left ventricular ejection fraction, septal and lateral E/E' ratios, and mitral regurgitation severity among the three groups. Figure 1 illustrates an example of LA size and LAEF measurements in three groups. The LAD was similar, but LAEF was $51 \%, 43 \%$, and $24 \%$ in the hearts of the no-AF, PAF, and non-PAF groups respectively. STE-derived LA function parameters, including LASr $(23.87 \% \pm 7.72 \%$,
$17.11 \% \pm 8.52 \%$, and $12.38 \% \pm 4.28 \%, \mathrm{P}<0.01)$, LASRr $\left(1.61 \pm 0.39,1.28 \pm 0.33\right.$, and $\left.1.05 \pm 0.24 \mathrm{~s}^{-1}, \mathrm{P}<0.01\right)$, and LASRa $\left(-1.75 \pm 0.54,-1.12 \pm 0.46\right.$, and $-0.70 \pm 0.28 \mathrm{~s}^{-1}$, $\mathrm{P}<0.01)$ were all significantly different among the no-AF, $\mathrm{PAF}$, and non-PAF groups. The post-hoc analyses show that LASr, LASRr, and LASRa were significantly different not only among the no-AF and AF groups but also between the PAF and non-PAF groups. For LASr, the $\mathrm{P}$ values were $<0.001$ between no-AF and PAF groups, and between non$\mathrm{AF}$ and non-PAF groups, and 0.009 between PAF and nonPAF groups; for LASRr, the $\mathrm{P}$ values were $<0.001$ between no-AF and PAF groups, and between non-AF and non-PAF groups, and 0.005 between PAF and non-PAF groups; for LASRa, the $\mathrm{P}$ values were all $<0.001$ between no-AF and PAF groups, between non-AF and non-PAF groups, and between PAF and non-PAF groups. Figure 2 illustrates a representative example of LA strain and strain rate curves and LASr, LASRr, LASRe, and LASRa measurements, 

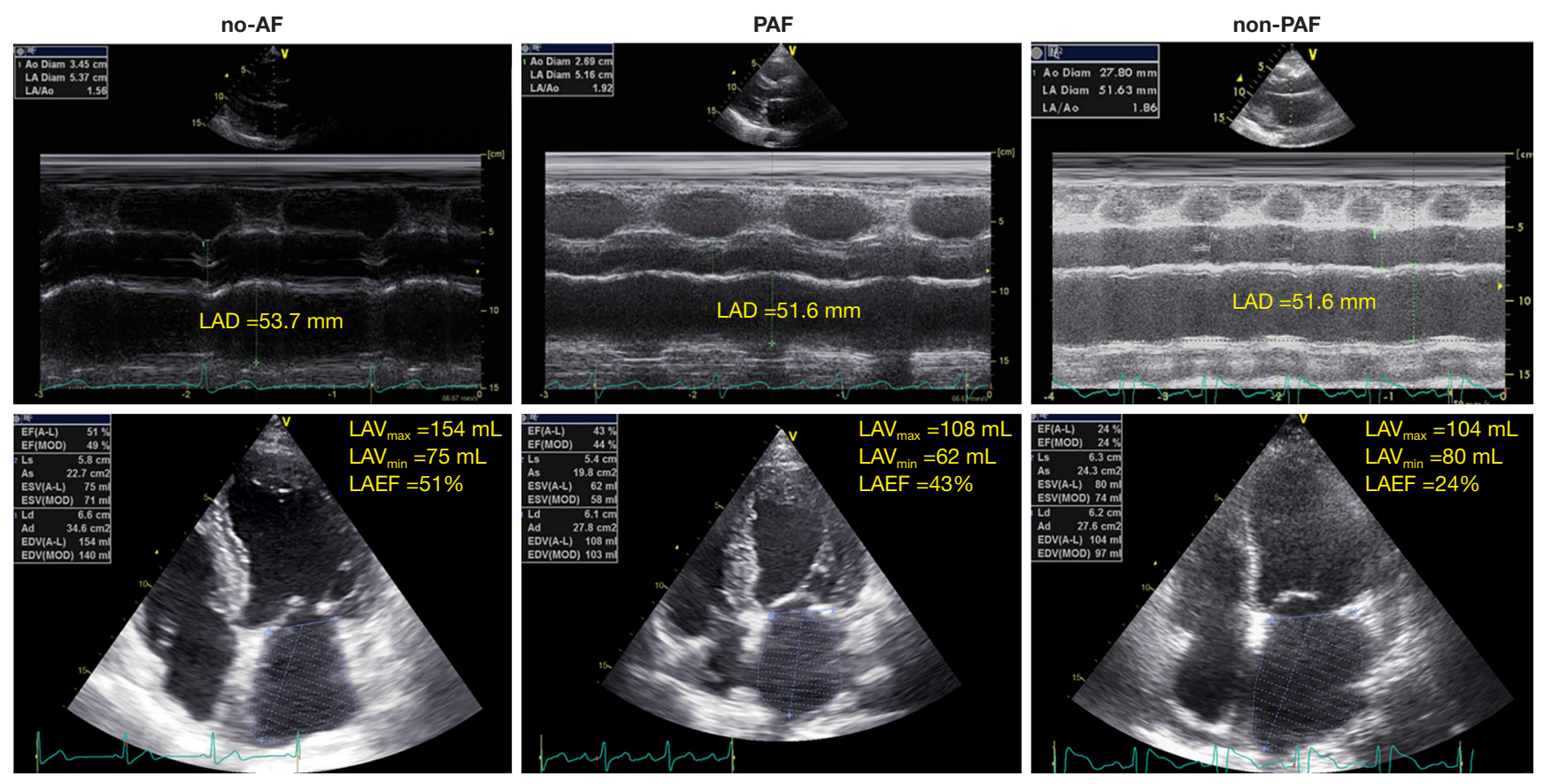

Figure 1 Representative left atrial (LA) dimension (LAD) in unidimensional measurement and the maximal LA volume (LAV $\mathrm{max}_{\text {ax }}$, minimal $\mathrm{LA}$ volume $\left(\mathrm{LAV}_{\min }\right)$, and LA emptying fraction $(\mathrm{LAEF})$ in 2-dimensional measurement in the no atrial fibrillation (no-AF), paroxysmal atrial fibrillation (PAF), and non-PAF groups.

which were highest in the no-AF group, followed by PAF and non-PAF groups.

To evaluate if RFCA significantly affected LA parameter measurement, we compared LA strain and strain rate among before ablation, 1-day and 3-month post ablation in 28 patients with PAF. The results showed that RFCA did not significantly influence LA strain and strain rate (Table S1), and additional LA linear ablation did not significantly influence LA strain and strain rate (Table S2) in patients with PAF. In the non-PAF group, we compared LA strain and strain rate between 1-day and 3-month post ablation in 26 patients who had no recurrence of $\mathrm{AF}$ at 3-month follow-up (Table S3). The results showed that the values of LA strain and strain rate were increased slightly 3 months after ablation, but these increases did not reach statistical significance.

\section{Associated factors of AF absence in patients with a severely dilated left atrium}

Table 3 illustrates the values of univariate and multivariate analyses. On univariate analysis, BMI, body surface area,
DM, hypertension, sick sinus syndrome, RHD, LAVImin, LAEF, E/A ratio, LASr, LASRr, and LASRa were significant factors associated with the presence of AF. However, the multivariate analysis showed that LASRa was the only significant factor associated with the presence of AF in patients with a severely dilated left atrium. The odds ratio was 21.69 and the $95 \%$ Ward confidence limit of LASRa was $9.77-48.13$.

\section{Reproducibility}

There was excellent reproducibility of STE analysis. The intraobserver and interobserver variabilities were 0.92 (95\% CI, 0.65-0.98) and 0.89 (95\% CI, 0.64-0.97), respectively, for LASr; 0.95 (95\% CI, 0.80-0.99) and 0.98 (95\% CI, $0.93-$ 1.00), respectively, for LASRr; 0.93 (95\% CI, 0.74-0.98) and 0.89 (95\% CI, 0.63-0.97), respectively, for LASRe; and 0.98 (95\% CI, 0.90-1.00) and 0.98 (95\% CI, 0.91-0.99), respectively, for LASRa. The reproducibility of STE values by analyzing two consecutive beats was also evaluated. As shown in the Table S4, the LA strain and strain rate values were not significantly different in these two beats. 

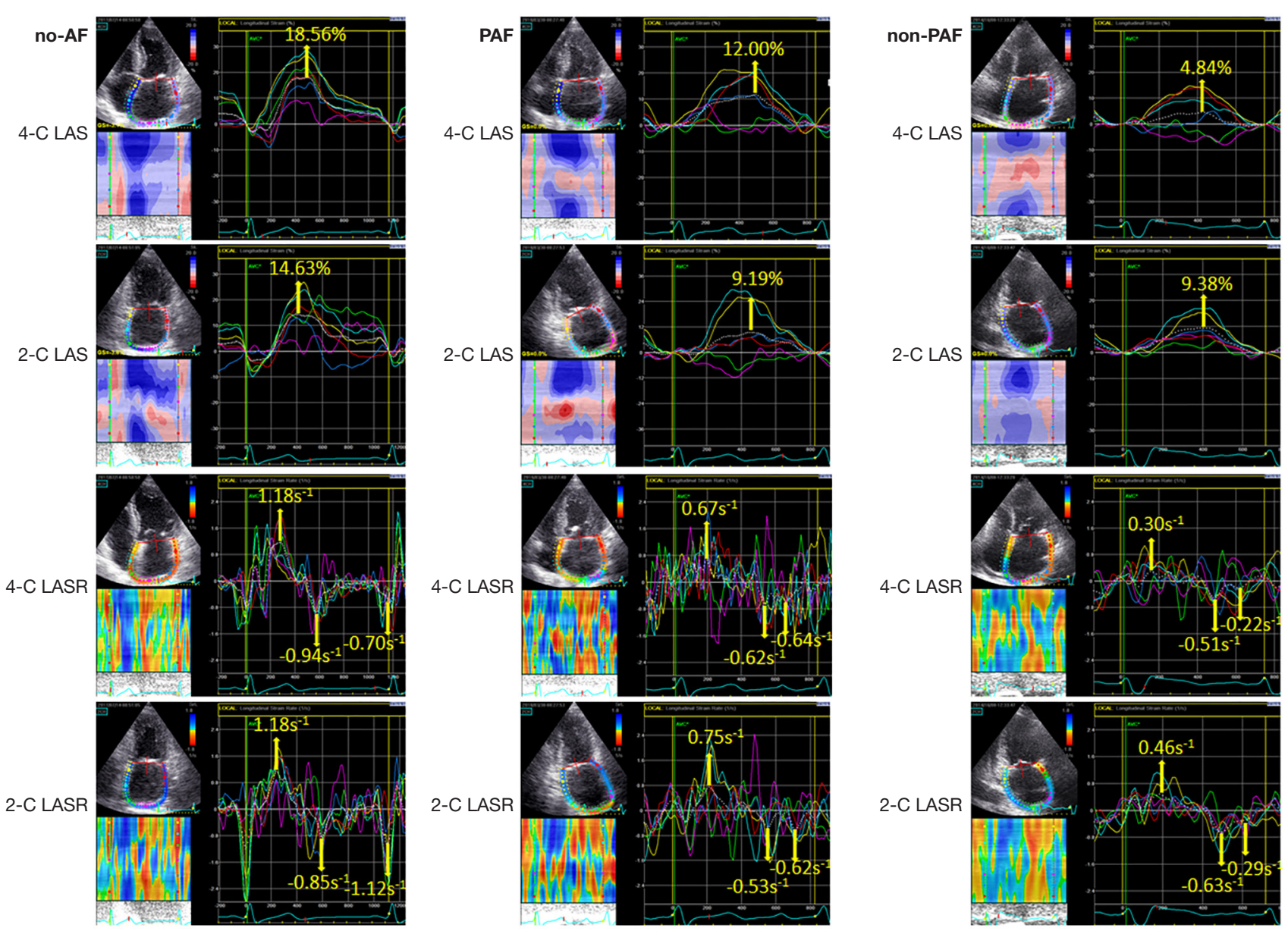

Figure 2 Representative left atrial strain (LAS) and strain rate (LASR) curves with measurements of mean LA peak longitudinal SR (LASr), systolic LASR (LASRr), early-diastolic LASR (LASRe), and late-diastoilc LASR (LASRa) in 4-chamber (4-C) and 2-chamber (2-C) views in the no atrial fibrillation (no-AF), paroxysmal atrial fibrillation (PAF), and non-PAF groups.

\section{Discussion}

In this retrospective case-control study, we found that LAVmin, LAEF, LASr, LASRr, and LASRa were significant echocardiographic associated factors and LASRa was the only independent factor associated with the presence of $\mathrm{AF}$ in patients with $\mathrm{LAD} \geq 50 \mathrm{~mm}$. Thus, LASRa, a site-specific measure of intrinsic atrial contractility, could help identify patients with a severely dilated left atrium at risk for AF. The no-AF group had higher prevalence rates of overweight, $\mathrm{DM}$, and hypertension that are closely interrelated conditions contributing to LA dilatation. This implies that these diseases may result in severe LA dilatation but with relatively preserved LA function. There are preliminary results suggesting that therapies such as weight reduction, angiotensin receptor blockers, aldosterone inhibitors, and statins may reduce atrial fibrosis to prevent and decrease $\mathrm{AF}$ burden (19-22). Pathological atrial enlargement should be prevented or reversed by addressing the initiating stimulus and disease (e.g., elevated pressure and volume) or targeting the key underlying mechanisms individually before more advanced atrial fibrosis and dysfunction occurs.

\section{$L A D \geq 50$ mm associated with $A F$, what else?}

In patients without a history of atrial arrhythmias or valvular heart disease, LA volume expressed the severity of diastolic dysfunction and provided an index of cardiovascular risk and disease burden (23). In the original Framingham cohort, LA size was the strongest independent predictor of new-onset $\mathrm{AF}$ (hazard ratio $=1.39 ; 95 \% \mathrm{CI}=1.14-1.68$ for 
Table 3 Univariate and multivariate analyses for the association with atrial fibrillation presence

\begin{tabular}{|c|c|c|c|c|c|c|}
\hline Variable & \multicolumn{3}{|c|}{ Univariate analysis } & \multicolumn{3}{|c|}{ Multivariate analysis } \\
\hline Body mass index $\left(\mathrm{kg} / \mathrm{m}^{2}\right)$ & 0.92 & $0.87-0.98$ & 0.01 & - & - & - \\
\hline Body surface area $\left(\mathrm{m}^{2}\right)$ & 0.22 & $0.05-0.88$ & 0.03 & - & - & - \\
\hline Diabetes mellitus & 0.42 & $0.21-0.83$ & 0.01 & - & - & - \\
\hline $\mathrm{RHD}$ & 3.51 & $1.25-9.84$ & 0.02 & - & - & - \\
\hline SSS & 5.899 & $1.71-20.32$ & 0.045 & - & - & - \\
\hline$L A V I_{\min }\left(m L / m^{2}\right)$ & 1.03 & $1.02-1.05$ & $<0.01$ & - & - & - \\
\hline LAEF (\%) & 0.94 & $0.92-0.96$ & $<0.01$ & - & - & - \\
\hline $\operatorname{LASRr}\left(\mathrm{S}^{-1}\right)$ & 0.03 & $0.01-0.09$ & $<0.01$ & - & - & - \\
\hline $\operatorname{LASRa}\left(\mathrm{S}^{-1}\right)$ & 21.05 & $9.54-46.47$ & $<0.01$ & 21.69 & $9.77-48.13$ & $<0.01$ \\
\hline
\end{tabular}

$\mathrm{Cl}$, confidence interval; LAVImin, minimal left atrial volume index; LAEF, left atrial emptying fraction; LASr, peak positive longitudinal strain during atrial filling; LASRr, strain rate during ventricular systole as an index for left atrial reservoir function; LASRa, peak negative contractile strain rates during left ventricular late refilling; RHD, rheumatic heart disease; SSS, sick sinus syndrome.

each $5 \mathrm{~mm}$ increase in the LAD) (3). In the general adult population, the risk of $\mathrm{AF}$ is proportionate to the degree of LA enlargement (24). The left atrium serves as a conduit between pulmonary veins and the left ventricle in diastole. In normal subjects, the LA function could be divided into three phases: reservoir, passive conduit, and pump phases, which accounted for around $40 \%, 35 \%$, and $25 \%$ of the atrial contribution of stroke volume, respectively (25). Abnormal LV relaxation increases left ventricular filling pressure, and thereby decreases passive emptying volume from the left atrium to the left ventricle which triggers a compensatory mechanism by enhancing active LA contraction to increase the active emptying volume in late diastole (24). While LA filling pressure is increased, atrial stretch leads to LA structural and electrophysiological remodeling, resulting in vulnerability to AF (26). In our cohort, all 160 patients had severely dilated LAD (52.8 \pm $2.6 \mathrm{~mm}$, range $50.0-62.6 \mathrm{~mm}$ ) and half of these patients developed AF. However, the other half of these patients did not have AF after a mean follow-up period of 30 months. This suggests that LA dilatation alone may not be enough for perpetuating $\mathrm{AF}$ and additional factors have to contribute to the occurrence of AF.

\section{Factors contributing to AF genesis}

Our study shows that the no-AF group had a higher prevalence of overweight, DM, and hypertension, which are closely interrelated conditions identified as independent risk markers for $\mathrm{AF}$ and may contribute to LA dilatation in these patients (27-30). However, all LA functional parameters, including LAEF, LASr, LASRr and LASRa, were better in the no-AF than the AF groups. This implies that a preserved LA function may counterbalance the arrhythmogenic effects of severely dilated left atrium to prohibit AF occurrence. According to the concept of Coumel's triangle of arrhythmogenesis: "There are always three main ingredients required for the production of a clinical arrhythmia: the arrhythmogenic substrate, the trigger factor and the modulation factors." (31). The majority of PAF is driven by focal sources from the thoracic veins (32), but non-PAF involves more complicated structural and electrophysiological remodeling. Specifically, AF is a multifactorial progressive disorder with different etiologies in different patient subpopulations. The potential interactions between LA dilatation and other mechanistic determinants of AF occurrence should be appreciated. It has been reported that atrial stretch $(33,34)$ and inflammation (35) 
contribute to electrical and structural remodeling and are implicated in the perpetuation of AF. There is also increasing evidence of microRNAs involvement in the pathogenesis of AF based on their link to fibrotic and apoptotic pathways (36). Atrial myopathy results from the development of fibrosis (7), which causes atrial dysfunction and localized regions of conduction slowing, thereby increasing AF susceptibility (37). Abhayaratna et al. reported that LA dysfunction represents a more advanced state of LA remodeling than LA enlargement alone to serve as a potent marker for new-onset AF or atrial flutter in persons $\geq 65$ years of age (38). Permanent sinus nodal dysfunction is commonly attributable to intrinsic factors, such as progressive fibrosis and ischemia (39). Whereas diffuse atrial myopathy providing a working substrate for $\mathrm{AF}$, bradycardia itself may also facilitate the development of AF through the increased likelihood of atrial ectopy and greater dispersion of refractoriness (40). Patients with RHD have sustained active inflammation that leads to not only valvular stenosis but also LA fibrosis (41), which is the best independent predictor of $\mathrm{AF}$ and seems to be a cause rather than an effect (42). These could explain why our data showed a higher prevalence of sick sinus syndrome and $\mathrm{RHD}$ in the AF groups than in the no-AF group.

\section{Inberent $L A$ contractility in predicting AF occurrence}

Petre et al. showed that LASr correlated with the onset of $\mathrm{AF}$ in patients with hypertension. The cut-off value of $19 \%$ of LASr had a sensitivity of $86 \%$ and a specificity of $77 \%$ (43). Consistently, our data shows that LASr was $24 \% \pm 8 \%$ in the no-AF group, $17 \% \pm 9 \%$ in the PAF group, and $12 \% \pm 4 \%$ in the non-PAF group. AF per se causes atrial electrophysiological remodeling, changes in connexin density and distribution, cellular structural remodeling, myolysis, and glycogen accumulation, leading to atrial wall stiffness (44), which progressively worsens as AF burden increases. It is noteworthy that multivariate analysis revealed that LASRa was the only independent associated factor of $\mathrm{AF}$ presence in patients with $\mathrm{LAD} \geq 50 \mathrm{~mm}$. Atrial pump function represents the inherent contractility of the LA myocardium, which is enhanced to compensate for a deceased atrial emptying volume when reservoir function is impaired and is highly effective in predicting the genesis and recurrence of AF $(45,46)$. Previous studies have shown that increased LV mass, abnormal geometry, and LV diastolic function are associated with impaired LA reservoir and conduit function in the presence of increased indexes of LA contractile function $(47,48)$. Therefore, a reduced LASRa implies a more advanced stage of diseased atrial myocardium than reduced LASRr and LASRe. Thomas et al. show that LASRa is a site-specific measure of intrinsic atrial contractility that is reduced in non-PAF after cardioversion to sinus rhythm (49). A temporal improvement is noted with the maintenance of sinus rhythm. However, a persisting degree of atrial dysfunction reflected by LASRa was still noted compared with the control healthy people despite the maintenance of sinus rhythm for a 6-month period, which warrants the longer-term use of antiarrhythmic treatment in the non-PAF cohort.

\section{Limitation}

Several limitations existed for the present study. First, this was a retrospective analysis with a small number in each group. The results of this study should be confirmed through further large-scale prospective studies. Second, the best study to evaluate the impact of clinical and echocardiographic factors on $\mathrm{AF}$ genesis is to evaluate if the LA STE parameters can predict a new-onset AF among those with enlarged LA and no previous history of AF. However, the event of a new-onset AF was zero in our noAF group during the 30 months of follow-up. We were not able to perform this kind of study. Third, even if our results showed that RFCA did not significantly influence LA strain and strain rate in the PAF or non-PAF groups, the number of patients in each group was small which would limit statistical accuracy and large-scale prospective studies are needed to clarify the influenced of RFCA on LA deformation properties in $\mathrm{AF}$ patients with severely dilated left atrium. Furthermore, the conclusion of this study cannot be applied to non-PAF patients with failed RFCA because we only collected the data when patients had sinus rhythm.

\section{Conclusions}

LA strain and strain rate are relatively preserved in patients with no $\mathrm{AF}$ compared with patients with $\mathrm{AF}$ even if the left atrium is severely dilated. Multivariate analysis revealed that LASRa is the only independent associated factor of $\mathrm{AF}$ presence in patients with $\mathrm{LAD} \geq 50 \mathrm{~mm}$. Compared with sick sinus syndrome and RHD, overweight, DM, and hypertension may result in severe LA dilatation but with relatively preserved LA function. Pathological atrial enlargement should be prevented by targeting the key 
underlying mechanisms individually (weight reduction and DM and hypertension control) before more advanced atrial fibrosis and dysfunction occurs.

\section{Acknowledgments}

Funding: None.

\section{Footnote}

Reporting Checklist: The authors have completed the STROBE reporting checklist. Available at https://qims. amegroups.com/article/view/10.21037/qims-21-954/rc

Conflicts of Interest: All authors have completed the ICMJE uniform disclosure form (available at https://qims. amegroups.com/article/view/10.21037/qims-21-954/coif). The authors have no conflicts of interest to declare.

Etbical Statement: The authors are accountable for all aspects of the work in ensuring that questions related to the accuracy or integrity of any part of the work are appropriately investigated and resolved. This study was conducted in accordance with the Declaration of Helsinki (as revised in 2013). This study was approved by the Institution Review Board of Chang Gung Memorial Hospital (No. 202101298B0) and individual consent for this retrospective analysis was waived.

Open Access Statement: This is an Open Access article distributed in accordance with the Creative Commons Attribution-NonCommercial-NoDerivs 4.0 International License (CC BY-NC-ND 4.0), which permits the noncommercial replication and distribution of the article with the strict proviso that no changes or edits are made and the original work is properly cited (including links to both the formal publication through the relevant DOI and the license). See: https://creativecommons.org/licenses/by-nc-nd/4.0/.

\section{References}

1. Hamatani Y, Ogawa H, Takabayashi K, Yamashita Y, Takagi D, Esato M, Chun YH, Tsuji H, Wada H, Hasegawa K, Abe M, Lip GY, Akao M. Left atrial enlargement is an independent predictor of stroke and systemic embolism in patients with non-valvular atrial fibrillation. Sci Rep 2016;6:31042.

2. Tsang TS, Barnes ME, Bailey KR, Leibson CL,
Montgomery SC, Takemoto Y, Diamond PM, Marra MA, Gersh BJ, Wiebers DO, Petty GW, Seward JB. Left atrial volume: important risk marker of incident atrial fibrillation in 1655 older men and women. Mayo Clin Proc 2001;76:467-75.

3. Vaziri SM, Larson MG, Benjamin EJ, Levy D. Echocardiographic predictors of nonrheumatic atrial fibrillation. The Framingham Heart Study. Circulation 1994;89:724-30.

4. Psaty BM, Manolio TA, Kuller LH, Kronmal RA, Cushman M, Fried LP, White R, Furberg CD, Rautaharju $\mathrm{PM}$. Incidence of and risk factors for atrial fibrillation in older adults. Circulation 1997;96:2455-61.

5. Bajraktari G, Bytyçi I, Henein MY. Left atrial structure and function predictors of recurrent fibrillation after catheter ablation: a systematic review and meta-analysis. Clin Physiol Funct Imaging 2020;40:1-13.

6. Burstein B, Nattel S. Atrial fibrosis: mechanisms and clinical relevance in atrial fibrillation. J Am Coll Cardiol 2008;51:802-9.

7. Rivner H, Mitrani RD, Goldberger JJ. Atrial Myopathy Underlying Atrial Fibrillation. Arrhythm Electrophysiol Rev 2020;9:61-70.

8. Lacalzada-Almeida J, García-Niebla J. How to detect atrial fibrosis. J Geriatr Cardiol 2017;14:185-94.

9. To AC, Flamm SD, Marwick TH, Klein AL. Clinical utility of multimodality LA imaging: assessment of size, function, and structure. JACC Cardiovasc Imaging 2011;4:788-98.

10. Patel RB, Delaney JA, Hu M, Patel H, Cheng J, Gottdiener J, Kizer JR, Marcus GM, Turakhia MP, Deo R, Heckbert SR, Psaty BM, Shah SJ. Characterization of cardiac mechanics and incident atrial fibrillation in participants of the Cardiovascular Health Study. JCI Insight 2020;5:141656.

11. Calkins H, Hindricks G, Cappato R, Kim YH, Saad EB, Aguinaga L, et al. 2017 HRS/EHRA/ECAS/APHRS/ SOLAECE expert consensus statement on catheter and surgical ablation of atrial fibrillation. Europace 2018;20:e1-e160.

12. Lang RM, Badano LP, Mor-Avi V, Afilalo J, Armstrong A, Ernande L, Flachskampf FA, Foster E, Goldstein SA, Kuznetsova T, Lancellotti P, Muraru D, Picard MH, Rietzschel ER, Rudski L, Spencer KT, Tsang W, Voigt JU. Recommendations for cardiac chamber quantification by echocardiography in adults: an update from the American Society of Echocardiography and the European Association of Cardiovascular Imaging. Eur Heart J 
Cardiovasc Imaging 2015;16:233-70.

13. Hwang YT, Lee HL, Lu CH, Chang PC, Wo HT, Liu HT, Wen MS, Lin FC, Chou CC. A Novel Approach for Predicting Atrial Fibrillation Recurrence After Ablation Using Deep Convolutional Neural Networks by Assessing Left Atrial Curved M-Mode Speckle-Tracking Images. Front Cardiovasc Med 2021;7:605642.

14. Mondillo S, Cameli M, Caputo ML, Lisi M, Palmerini E, Padeletti M, Ballo P. Early detection of left atrial strain abnormalities by speckle-tracking in hypertensive and diabetic patients with normal left atrial size. J Am Soc Echocardiogr 2011;24:898-908.

15. Montserrat S, Gabrielli L, Bijnens B, Borràs R, Berruezo A, Poyatos S, Brugada J, Mont L, Sitges M. Left atrial deformation predicts success of first and second percutaneous atrial fibrillation ablation. Heart Rhythm 2015;12:11-8.

16. Yang CH, Chou CC, Hung KC, Wen MS, Chang PC, Wo HT, Lee CH, Lin FC. Comparisons of the underlying mechanisms of left atrial remodeling after repeat circumferential pulmonary vein isolation with or without additional left atrial linear ablation in patients with recurrent atrial fibrillation. Int J Cardiol 2017;228:449-55.

17. Liu HT, Yang CH, Lee HL, Chang PC, Wo HT, Wen MS, Wang CC, Yeh SJ, Chou CC. Clinical Outcomes of low-voltage area-guided left atrial linear ablation for non-paroxysmal atrial fibrillation patients. PLoS One 2021;16:e0260834.

18. Chou CC, Lee HL, Chang PC, Wo HT, Wen MS, Yeh SJ, Lin FC, Hwang YT. Left atrial emptying fraction predicts recurrence of atrial fibrillation after radiofrequency catheter ablation. PLoS One 2018;13:e0191196.

19. Schneider MP, Hua TA, Böhm M, Wachtell K, Kjeldsen SE, Schmieder RE. Prevention of atrial fibrillation by Renin-Angiotensin system inhibition a meta-analysis. J Am Coll Cardiol 2010;55:2299-307.

20. Dąbrowski R, Szwed H. Antiarrhythmic potential of aldosterone antagonists in atrial fibrillation. Cardiol J 2012;19:223-9.

21. Fauchier L, Clementy N, Babuty D. Statin therapy and atrial fibrillation: systematic review and updated metaanalysis of published randomized controlled trials. Curr Opin Cardiol 2013;28:7-18.

22. Nalliah CJ, Sanders P, Kottkamp H, Kalman JM. The role of obesity in atrial fibrillation. Eur Heart J 2016;37:1565-72.

23. Tsang TS, Barnes ME, Gersh BJ, Bailey KR, Seward JB. Left atrial volume as a morphophysiologic expression of left ventricular diastolic dysfunction and relation to cardiovascular risk burden. Am J Cardiol 2002;90:1284-9.

24. Tiwari S, Schirmer H, Jacobsen BK, Hopstock LA, Nyrnes A, Heggelund G, Njølstad I, Mathiesen EB, Løchen ML. Association between diastolic dysfunction and future atrial fibrillation in the Tromsø Study from 1994 to 2010 . Heart 2015;101:1302-8.

25. Todaro MC, Choudhuri I, Belohlavek M, Jahangir A, Carerj S, Oreto L, Khandheria BK. New echocardiographic techniques for evaluation of left atrial mechanics. Eur Heart J Cardiovasc Imaging 2012;13:973-84.

26. Jaïs P, Peng JT, Shah DC, Garrigue S, Hocini M, Yamane T, Haïssaguerre M, Barold SS, Roudaut R, Clémenty J. Left ventricular diastolic dysfunction in patients with socalled lone atrial fibrillation. J Cardiovasc Electrophysiol 2000;11:623-5.

27. Abed HS, Wittert GA, Leong DP, Shirazi MG, Bahrami B, Middeldorp ME, Lorimer MF, Lau DH, Antic NA, Brooks AG, Abhayaratna WP, Kalman JM, Sanders P. Effect of weight reduction and cardiometabolic risk factor management on symptom burden and severity in patients with atrial fibrillation: a randomized clinical trial. JAMA 2013;310:2050-60.

28. Tsang TS, Barnes ME, Miyasaka Y, Cha SS, Bailey KR, Verzosa GC, Seward JB, Gersh BJ. Obesity as a risk factor for the progression of paroxysmal to permanent atrial fibrillation: a longitudinal cohort study of 21 years. Eur Heart J 2008;29:2227-33.

29. Kannel WB, Wolf PA, Benjamin EJ, Levy D. Prevalence, incidence, prognosis, and predisposing conditions for atrial fibrillation: population-based estimates. Am J Cardiol 1998;82:2N-9N.

30. Benjamin EJ, Levy D, Vaziri SM, D'Agostino RB, Belanger AJ, Wolf PA. Independent risk factors for atrial fibrillation in a population-based cohort. The Framingham Heart Study. JAMA 1994;271:840-4.

31. Farré J, Wellens HJ. Philippe Coumel: a founding father of modern arrhythmology. Europace 2004;6:464-5.

32. Chen PS, Chou CC, Tan AY, Zhou S, Fishbein MC, Hwang C, Karagueuzian HS, Lin SF. The mechanisms of atrial fibrillation. J Cardiovasc Electrophysiol 2006;17 Suppl 3:S2-7.

33. Ninio DM, Saint DA. The role of stretch-activated channels in atrial fibrillation and the impact of intracellular acidosis. Prog Biophys Mol Biol 2008;97:401-16.

34. Li D, Fareh S, Leung TK, Nattel S. Promotion of atrial fibrillation by heart failure in dogs: atrial remodeling of a 
different sort. Circulation 1999;100:87-95.

35. Van Wagoner DR. Oxidative stress and inflammation in atrial fibrillation: role in pathogenesis and potential as a therapeutic target. J Cardiovasc Pharmacol 2008;52:306-13.

36. Wang Z, Lu Y, Yang B. MicroRNAs and atrial fibrillation: new fundamentals. Cardiovasc Res 2011;89:710-21.

37. Thomas L, Abhayaratna WP. Left Atrial Reverse Remodeling: Mechanisms, Evaluation, and Clinical Significance. JACC Cardiovasc Imaging 2017;10:65-77.

38. Abhayaratna WP, Fatema K, Barnes ME, Seward JB, Gersh BJ, Bailey KR, Casaclang-Verzosa G, Tsang TS. Left atrial reservoir function as a potent marker for first atrial fibrillation or flutter in persons $>$ or $=65$ years of age. Am J Cardiol 2008;101:1626-9.

39. John RM, Kumar S. Sinus Node and Atrial Arrhythmias. Circulation 2016;133:1892-900.

40. Amasyali B, Kilic A, Kilit C. Sinus node dysfunction and atrial fibrillation: which one dominates? Int J Cardiol 2014;175:379-80.

41. Shiba M, Sugano Y, Ikeda Y, Okada H, Nagai T, IshibashiUeda H, Yasuda S, Ogawa H, Anzai T. Presence of increased inflammatory infiltrates accompanied by activated dendritic cells in the left atrium in rheumatic heart disease. PLoS One 2018;13:e0203756.

42. Ismail AS, Baghdady Y, Salem MA, Wahab AA. The use of MRI in quantification of the atrial fibrosis in patients with rheumatic mitral disease. Egyptian Journal of Radiology abd Nuclear Medicine 2020;51:1-10.

43. Petre I, Onciul S, Iancovici S, Zamfir D, Stoian M, Scărlătescu A, Diaconeasa A, Acatrinei C, Dorobanțu M. Left Atrial Strain for Predicting Atrial Fibrillation Onset

Cite this article as: Yang CH, Liu HT, Lee HL, Lin FC, Chou CC. Left atrial booster-pump function as a predictive parameter for atrial fibrillation in patients with severely dilated left atrium. Quant Imaging Med Surg 2022;12(4):2523-2534. doi: 10.21037/qims-21-954 in Hypertensive Patients. High Blood Press Cardiovasc Prev 2019;26:331-7.

44. Ausma J, Wijffels M, Thoné F, Wouters L, Allessie M, Borgers M. Structural changes of atrial myocardium due to sustained atrial fibrillation in the goat. Circulation 1997;96:3157-63.

45. Shang Z, Su D, Cong T, Sun Y, Liu Y, Chen N, Yang J. Assessment of left atrial mechanical function and synchrony in paroxysmal atrial fibrillation with twodimensional speckle tracking echocardiography. Echocardiography 2017;34:176-83.

46. Fatema K, Barnes ME, Bailey KR, Abhayaratna WP, Cha S, Seward JB, Tsang TS. Minimum vs. maximum left atrial volume for prediction of first atrial fibrillation or flutter in an elderly cohort: a prospective study. Eur J Echocardiogr 2009;10:282-6.

47. Wang Z, Tan H, Zhong M, Jiang G, Zhang Y, Zhang W. Strain rate imaging for noninvasive functional quantification of the left atrium in hypertensive patients with paroxysmal atrial fibrillation. Cardiology 2008;109:15-24.

48. Kokubu N, Yuda S, Tsuchihashi K, Hashimoto A, Nakata T, Miura T, Ura N, Nagao K, Tsuzuki M, Wakabayashi C, Shimamoto K. Noninvasive assessment of left atrial function by strain rate imaging in patients with hypertension: a possible beneficial effect of reninangiotensin system inhibition on left atrial function. Hypertens Res 2007;30:13-21.

49. Thomas L, McKay T, Byth K, Marwick TH. Abnormalities of left atrial function after cardioversion: an atrial strain rate study. Heart 2007;93:89-95. 


\section{Supplementary}

Table S1 Comparisons of mechanical deformation parameters before ablation, 1-day and 3-month post ablation in the paroxysmal atrial fibrillation group

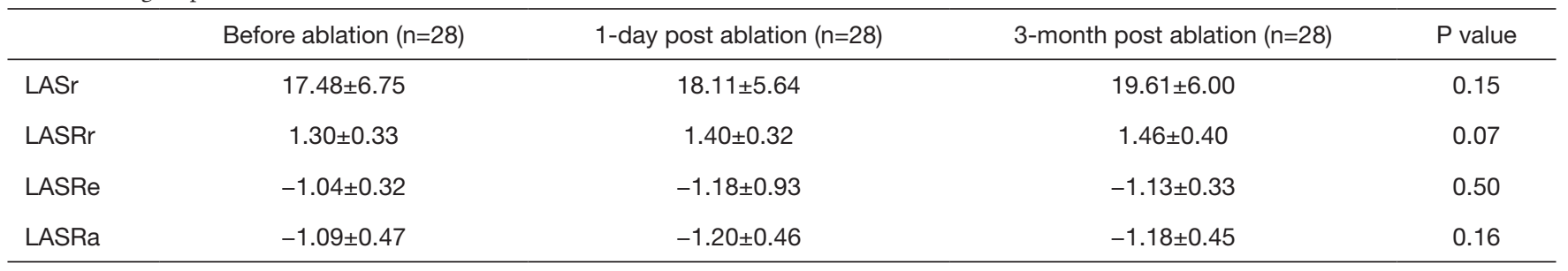

LASr, peak positive longitudinal strain during atrial filling; LASRr, strain rate during ventricular systole as an index for left atrial reservoir function; LASRe and LASRa, peak negative conduit and contractile strain rates during left ventricular early and late refilling, respectively.

Table S2 Comparisons of mechanical deformation parameters before ablation, 1-day and 3-month post ablation in patients with and without additional left atrial linear ablation (LARF) in the paroxysmal atrial fibrillation group

\begin{tabular}{llcccc}
\hline & & Before ablation & 1-day post ablation & 3-month post ablation & P value \\
\hline \multirow{2}{*}{ LASr } & LARF $(+)(n=14)$ & $17.61 \pm 7.02$ & $17.69 \pm 4.12$ & $19.78 \pm 5.70$ & 0.44 \\
\multirow{2}{*}{ LASRr } & LARF $(-)(n=14)$ & $17.35 \pm 6.74$ & $18.52 \pm 6.98$ & $19.43 \pm 6.49$ & 0.12 \\
& LARF $(+)(n=14)$ & $1.28 \pm 0.23$ & $1.38 \pm 0.17$ & $1.51 \pm 0.33$ & 0.08 \\
\multirow{2}{*}{ LASRe } & LARF $(-)(n=14)$ & $1.32 \pm 0.41$ & $1.42 \pm 0.43$ & $-1.41 \pm 0.46$ & 0.36 \\
& LARF $(+)(n=14)$ & $-1.10 \pm 0.29$ & $-1.07 \pm 0.34$ & $-1.24 \pm 0.36$ & 0.17 \\
LASRa & LARF $(-)(n=14)$ & $-0.97 \pm 0.34$ & $-1.30 \pm 1.29$ & $-1.01 \pm 0.25$ & 0.36 \\
& LARF $(+)(n=14)$ & $-1.10 \pm 0.43$ & $-1.19 \pm 0.36$ & $-1.16 \pm 0.28$ & 0.53 \\
\hline
\end{tabular}

LASr, peak positive longitudinal strain during atrial filling; LASRr, strain rate during ventricular systole as an index for left atrial reservoir function; LASRe and LASRa, peak negative conduit and contractile strain rates during left ventricular early and late refilling, respectively.

Table S3 Comparisons of mechanical deformation parameters 1-day and 3-month post ablation in the non-paroxysmal atrial fibrillation group

\begin{tabular}{lccc}
\hline & 1-day post ablation $(\mathrm{n}=26)$ & 3-month post ablation $(\mathrm{n}=26)$ & $\mathrm{P}$ value \\
\hline LASr & $12.50 \pm 4.32$ & $13.16 \pm 5.15$ & 0.22 \\
LASRr & $1.05 \pm 0.24$ & $1.06 \pm 0.7$ & 0.90 \\
LASRe & $-1.02 \pm 0.37$ & $-1.07 \pm 0.40$ & 0.23 \\
LASRa & $-0.71 \pm 0.27$ & $-0.74 \pm 0.37$ & 0.64 \\
\hline
\end{tabular}

LASr, peak positive longitudinal strain during atrial filling; LASRr, strain rate during ventricular systole as an index for left atrial reservoir function; LASRe and LASRa, peak negative conduit and contractile strain rates during left ventricular early and late refilling, respectively.

Table S4 Comparisons of the beat-to-beat difference when study group in sinus rhythm

\begin{tabular}{lccc}
\hline & Cycle 1 $(\mathrm{n}=5)$ & Cycle 2 $(\mathrm{n}=5)$ & P value \\
\hline LASr $(\%)$ & $19.84 \pm 7.48$ & $19.78 \pm 8.26$ & 0.95 \\
LASRr $\left(\mathrm{S}^{-1}\right)$ & $1.33 \pm 0.32$ & $1.24 \pm 0.24$ & 0.30 \\
LASRe $\left(\mathrm{S}^{-1}\right)$ & $-1.24 \pm 0.33$ & $-1.13 \pm 0.36$ & 0.37 \\
LASRa $\left(\mathrm{S}^{-1}\right)$ & $-1.04 \pm 0.66$ & $-1.05 \pm 0.71$ & 0.92 \\
\hline
\end{tabular}

LASr, peak positive longitudinal strain during atrial filling; LASRr, strain rate during ventricular systole as an index for left atrial reservoir function; LASRe and LASRa, peak negative conduit and contractile strain rates during left ventricular early and late refilling, respectively. 